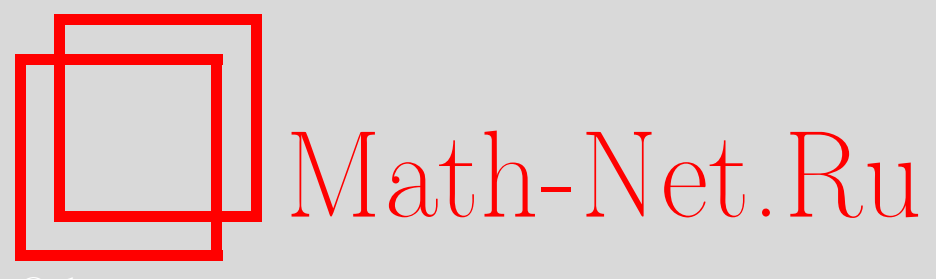

В. В. Борисенко, О трансверсалях распавшихся латинских квадратов четного порядка, Матем. вопр. криптогр., 2014, том 5, выпуск 1, 5-25

DOI: https://doi.org/10.4213/mvk104

Использование Общероссийского математического портала Math-Net.Ru подразумевает, что вы прочитали и согласны с пользовательским соглашением http: //www . mathnet.ru/rus/agreement

Параметры загрузки:

IP: 54.209 .52 .79

26 апреля 2023 г., 14:50:42

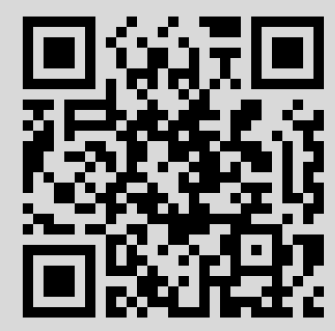




\author{
МАТЕМАТИЧЕСКИЕ ВОПРОСЫ КРИПТОГРАФИИ \\ 2014 T. 5 № 1 C. 5-25
}

УДК: 519.143

\title{
О трансверсалях распавшихся латинских квадратов четного порядка
}

\author{
В. В. Борисенко \\ ООО «Центр сертификационных исследований», г. Москва
}

Получено 22.IV.2013

Рассматриваются распавшиеся латинские квадраты, т. е. латинские квадраты порядка $2 n$, построенные на множестве чисел $\{0, \ldots, 2 n-1\}$, которые при приведении по модулю $n$ дают $2 n \times 2 n$-матрицу, состоящую из четырех латинских квадратов порядка $n$. Множество всех трансверсалей распавшегося латинского квадрата описано с помощью 2-сбалансированных мультимножеств позиций одного из упомянутых выше латинских квадратов порядка $n$. Приведен алгоритм, позволяющий (после некоторой предварительной работы) быстро строить множество трансверсалей для любого распавшегося латинского квадрата порядка $2 n$, отвечающего произвольной четверке латинских квадратов порядка $n$.

Ключевые слова: латинский квадрат, трансверсаль, мультимножество

\section{Transversals in splitted Latin squares of even order}

\section{V. Borisenko}

LLC "Sertification Research Center", Moscow

\begin{abstract}
We consider the splitted Latin squares, i. e. Latin squares of order $2 n$ with elements from $\{0, \ldots, 2 n-1\}$ such that after reducing modulo $n$ we obtain $2 n \times 2 n$-matrix consisting of four Latin squares of order $n$. The set of all transversals of splitted Latin square is described by means of 2-balansed multisets of entries of one of Latin squares of order $n$ mentioned above. A quick algorithm of construction (after some preliminary work) the set of all transversals for any splitted Latin square of order $2 n$ corresponding to an arbitrary set of four Latin squares of order $n$ is described.
\end{abstract}

Key words: Latin square, transversal, multise

Citation: Mathematical Aspects of Cryptography, 2014, vol. 5, no. 1, pp. 5-25 (Russian). 


\section{1. Введение}

Латинским квадратом (далее для краткости - ЛК) порядка $n \in \mathbb{N}$ на множестве $M=\left\{m_{1}, \ldots, m_{n}\right\}$ называется квадратная матрица $A=\left(a_{i j}\right)_{n \times n}$, в которой все строки и столбцы являются перестановками элементов множества $M$. Далее всюду без ограничения общности будем полагать, что $M=\{0,1, \ldots, n-1\}$, и элементы этого множества при необходимости будут рассматриваться как наименьшие неотрицательные вычеты по модулю $n$.

Упорядоченную пару $(i, j) \in\{1, \ldots, n\}^{2}$ будем рассматривать как позицию в ЛК $A$, а произвольное подмножество $\lambda \subset\{1, \ldots, n\}^{2}-$ как множество позиций в этом квадрате. Множества позиций вида $\rho_{s}=$ $=\{(s, j) \mid j \in\{1, \ldots, n\}\}$ и $\xi_{t}=\{(i, t) \quad \mid i \in\{1, \ldots, n\}\}$ называются $s$-й строкой и $t$-м столбцом ЛК $A$ соответственно.

Подстановка $\alpha$ степени $n$ задает в ЛК $A$ множество позиций вида $\{(i, \alpha(i)) \mid i \in\{1, \ldots, n\}\}$, которое в данной работе для простоты будем также называть подстановкой и отождествлять с $\alpha$. Для произвольного $a \in$ $\in\{0, \ldots, n-1\}$ множество позиций $\left\{(i, j) \mid a_{i j}=a\right\}$ является подстановкой и называется диагональю ЛК $A$ с элементом $a$. Всего в любом ЛК порядка $n$ имеется ровно $n$ диагоналей, по одной для каждого $a \in\{0, \ldots, n-1\}$. Подстановка $\alpha \subset\{1, \ldots, n\}^{2}$ называется трансверсалью ЛК $A$, если $\left\{a_{i j} \mid\right.$ $\mid(i, j) \in \alpha\}=\{0, \ldots, n-1\}$, т. е. на позициях $\alpha$ встречаются все элементы из $\{0, \ldots, n-1\}$.

Обобщением понятия трансверсали в латинском квадрате является понятие $k$-трансверсали (или $k$-плекса, см. [2, 3]).

Определение. Множество позиций $\tau \subset\{1, \ldots, n\}^{2}$ называется $k$-трансверсалью ЛК $A, k \in\{1, \ldots, n\}$, если $\tau$ имеет в каждой строке и каждом столбце ровно по $k$ позиций и каждый элемент $a \in\{0, \ldots, n-1\}$ встречается в ЛК $A$ на позициях $\tau$ ровно $k$ раз.

Очевидно, что понятия обычной трансверсали и 1-трансверсали совпадают. Множество всех $k$-трансверсалей ЛК $A$ будем обозначать через $\operatorname{Tr}_{k}(A)$ и при $k=1$ индекс опускать: $\operatorname{Tr}_{1}(A)=\operatorname{Tr}(A)$.

С произвольным множеством позиций $\lambda \subset\{1, \ldots, n\}^{2}$ будем связывать $(0,1)$-матрицу $S=\left(s_{i j}\right)_{n \times n}$ порядка $n$, в которой $s_{i j}=1 \Leftrightarrow(i, j) \in \lambda$, т. е. единицы указывают позиции, принадлежащие $\lambda$. В данной работе будет использоваться операция поэлементного произведения целочисленных матриц одинакового порядка: $U=S * T \Leftrightarrow u_{i j}=s_{i j} t_{i j} \forall(i, j) \in\{1, \ldots, n\}^{2}$.

Преобразование ЛК $A$, состоящее в перестановке его строк, столбцов и взаимно однозначной замене его элементов, называется изотопией. Если 
при некоторой изотопии ЛК $A$ преобразуется в ЛК $B$, то такие квадраты называются изотопными. Изотопность является эквивалентностью на множестве всех ЛК одного порядка, заданных на одном и том же множестве элементов M. Изотопия называется главной, если она представляет собой только замену элементов квадрата без перестановки его строк или столбцов. Очевидно, что если ЛК $A$ и $B$ изотопны, то определена биекция между множествами $\operatorname{Tr}_{k}(A)$ и $\operatorname{Tr}_{k}(B)$, а если эти квадраты главно изотопны, то $\operatorname{Tr}_{k}(A)=\operatorname{Tr}_{k}(B)$.

Описание множеств трансверсалей для ЛК, относящихся к тем или иным классам, является актуальной задачей с точки зрения построения систем ортогональных латинских квадратов.

ЛК $A$ и $B$ одинакового порядка $n$ на множестве $\{0, \ldots, n-1\}$ называются ортогональными, если при их наложении друг на друга получается ровно $n^{2}$ различных упорядоченных пар $\left(a_{i j}, b_{i j}\right),(i, j) \in\{1, \ldots, n\}^{2}$. В этом случае будем писать $A \perp B$. Набор ЛК $\mathcal{L}=\left\{A_{1}, \ldots, A_{t}\right\}$ называется системой попарно ортогональных латинских квадратов (сокращенно - СОЛК), если $A_{s} \perp A_{r}$ при любых $s \neq r$.

СОЛК $\mathcal{L}$ называется максимальной, если ее нельзя расширить присоединением нового квадрата, и полной, если $|\mathcal{L}|=t=n-1$ (большего числа квадратов в СОЛК быть не может, см. [1, с. 389]). Следующее легко доказываемое утверждение дает критерий того, что ЛК $A$ входит в некоторую СОЛК, состоящую из $t$ квадратов.

Утверждение 0. ЛК А порядка п входит в СОЛК из $t$ квадратов в том и только том случае, когда имеется $t-1$ наборов $T_{1}, \ldots T_{t-1}$, состоящих из $n$ попарно не пересекаюшихся трансверсалей ЛК А каждый, причем любые две трансверсали из разных наборов пересекаются ровно по одному элементу.

Обычно это утверждение приводится для $t=2$ как критерий наличия у ЛК $A$ ортогональной пары $B$. В работе [4] (теорема 7) оно сформулировано и доказано при $t=3$ как критерий вхождения ЛК $A$ в СОЛК из трех квадратов. В общем виде это утверждение автору в литературе не встретилось, что, по-видимому, объясняется очевидностью его доказательства: указанные наборы трансверсалей $T_{1}, \ldots, T_{t-1}$ ЛК $A$ являются соответственно наборами диагоналей ЛК $A_{1}, \ldots, A_{t-1}$, взаимно ортогональных и ортогональных $A$.

Если теперь к семейству наборов $\left\{T_{1}, \ldots, T_{t-1}\right\}$ добавить все строки $R=\left\{\rho_{1}, \ldots, \rho_{n}\right\}$, столбцы $C=\left\{\xi_{1}, \ldots, \xi_{n}\right\}$ и диагонали $D=\left\{\delta_{1}, \ldots, \delta_{n}\right\}$ ЛК $A$, то получится семейство наборов $\left\{R, C, D, T_{1}, \ldots, T_{t-1}\right\}$, которое естественно рассматривать как конечную геометрию $\mathcal{G}=(\mathcal{P}, \mathcal{S})$ со множеством точек $\mathcal{P}=\{1, \ldots, n\}^{2}$ и линий $\mathcal{S}=R \cup C \cup D \cup \bigcup_{i=1}^{t-1} T_{i}$. Тогда получается, 
что всего линий ровно $(t+2) n$, на каждой линии имеется ровно $n$ точек, множество линий разбито на $t+2$ пучков параллельных линий, и любые две линии из различных пучков имеют ровно одну общую точку. При $t=n-1$ эта геометрия представляет собой конечную аффинную плоскость порядка $n$. При этом же условии СОЛК $\mathcal{L}=\left\{A=A_{0}, A_{1}, \ldots, A_{t-1}\right\}$ является полной. Этот факт равносилен известной теореме Боуза о том, что полная СОЛК, состоящая из квадратов порядка $n$, существует тогда и только тогда, когда существует конечная проективная плоскость порядка $n$ (см. [1, с. 393]).

Приведенные рассуждения указывают (по мнению автора) на более естественную связь полных СОЛК с конечными аффинными плоскостями, чем с конечными проективными плоскостями. Вся геометрия конечной аффинной плоскости уже имеется в ЛК $A$, входящем в полную СОЛК. Для получения же из нее конечной проективной плоскости в соответствии с известной стандартной процедурой необходимо добавление $n+1$ несобственных точек и одной несобственной прямой, которые не имеют естественной интерпретации в ЛК $A$.

Таким образом, при решении задачи о том, входит ли заданный ЛК $A$ в какую-нибудь СОЛК, прежде всего требуется построить все его трансверсали. Затем возникает задача о существовании во множестве $\operatorname{Tr}(A)$ системы подмножеств $\left\{T_{1}, \ldots, T_{t-1}\right\}$ с указанными в утверждении 0 свойствами, или, что то же самое, задача о существовании в $\operatorname{Tr}(A)$ описанной выше конечной геометрии $\mathcal{G}=(\mathcal{P}, \mathcal{S})$.

Целью настоящей работы является описание множеств трансверсалей для латинских квадратов, относящихся к определяемому ниже классу распавшихся ЛК.

\section{2. Распавшиеся латинские квадраты и их основные свойства}

Определение. ЛК $F=\left(f_{i j}\right)_{2 n \times 2 n}$ порядка $2 n$ на множестве $\{0, \ldots$, $2 n-1\}$ будем называть распавшимся, если после приведения его элементов по модулю $n$ из него получается матрица $G=\left(\begin{array}{ll}A & C \\ B & D\end{array}\right)=\left(g_{i j}\right)_{2 n \times 2 n}$, в которой матрицы $A, B, C, D$ порядка $n$ являются латинскими квадратами. Если при этом $A=B=C=D$, то ЛК $F$ будем называть однородным.

По распавшемуся ЛК $F$ построим $(0,1)$-матрицу $T_{F}=\left(t_{i j}\right)_{2 n \times 2 n}$ порядка $2 n$, в которой элемент $t_{i j}=1 \Leftrightarrow f_{i j} \in\{0, \ldots, n-1\}$. Разобьем матрицу $T_{F}$ на 4 матрицы порядка $n$ аналогично разбиению матрицы $G: T_{F}=\left(\begin{array}{c}T_{A} T_{C} \\ T_{B}\end{array} T_{D}\right)$. Очевидно, что по набору латинских квадратов и $(0,1)$-матриц порядка $n$ : 
$\left(A, B, C, D, T_{A}, T_{B}, T_{C}, T_{D}\right)$ однозначно восстанавливается исходный распавшийся ЛК $F$. Поэтому будем говорить, что распавшийся ЛК $F$ задан набором ЛК $(A, B, C, D)$ и трафаретов $\left(T_{A}, T_{B}, T_{C}, T_{D}\right)$, и обозначать этот факт равенством $F=\left(A, B, C, D, T_{A}, T_{B}, T_{C}, T_{D}\right)$.

Рассмотрим вопрос о том, сколько распавшихся ЛК порядка $2 n$ можно построить, исходя из заданного набора $(A, B, C, D)$ латинских квадратов порядка $n$, изменяя всевозможными допустимыми способами набор трафаретов $\left(T_{A}, T_{B}, T_{C}, T_{D}\right)$.

Определение. а) Пусть $A, B$ - два ЛК порядка $n$ на множестве $\{0, \ldots, n-1\}$. Будем говорить, что они задают строчную подстановку $\sigma_{A B}$ степени $n^{2}$, действующую на множестве $\{1, \ldots, n\}^{2}$, определяемую следующим образом:

$$
\sigma_{A B}(i, j)=(i, k) \Leftrightarrow a_{i j}=b_{i k}
$$

Действие подстановки $\sigma_{A B}$ изображено в виде схемы на рисунке 1 .

Здесь ЛК $A, B$ «состыкованы» построчно, и $\sigma_{A B}$ переводит позицию $(i, j)$ в позицию $(i, k)$ в случае совпадения элементов $a_{i j}=b_{i k}$.

б) Аналогично два ЛК $A, B$ порядка $n$ на множестве $\{0, \ldots, n-1\}$ определяют столбцовую подстановку $\kappa_{A B}$ степени $n^{2}$, действующую на множестве $\{1, \ldots, n\}^{2}$, следующим об-

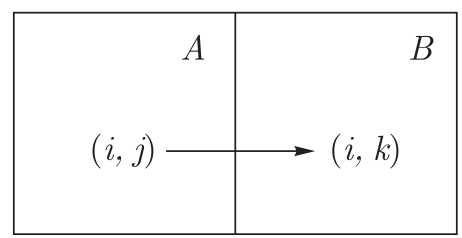

Рис. 1 разом: $\kappa_{A B}(i, j)=(k, j) \Leftrightarrow a_{i j}=b_{k j}$. Действие $\kappa_{A B}$ реализуется по схеме, изображенной на рисунке 2 .

Здесь ЛК $A, B$ «состыкованы» по столбцам, и $\kappa_{A B}$ переводит позицию $(i, j)$ в позицию $(k, j)$ в случае совпадения элементов $a_{i j}=b_{k j}$.

в) Пусть $A, B, C, D-4$ ЛК порядка $n$ на множестве $\{0, \ldots, n-1\}$. Они определяют действующую на множестве $\{1, \ldots, n\}^{2}$ подстановку $\chi_{A C D B}$ степени $n^{2}$ как произведение строчных и столбцовых подстановок $\chi_{A C D B}=$ $=\sigma_{A C} \kappa_{C D} \sigma_{D B} \kappa_{B A}$, применяемых слева направо, и ей соответствует схема на рисунке 3 , где $\chi_{A C D B}(i, j)=(l, t)$.

ЗАМЕЧАНИЕ. Очевидно, что при действии строчных и столбцовых подстановок диагонали латинских квадратов переходят в диагонали. Поэтому циклы подстановки $\chi_{A C D B}$ являются подмножествами диагоналей ЛК $A$. 


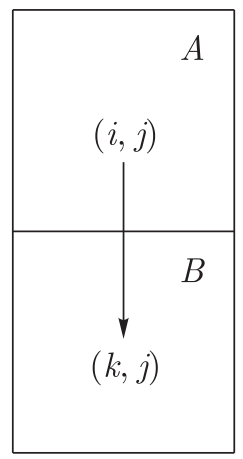

Рис. 2

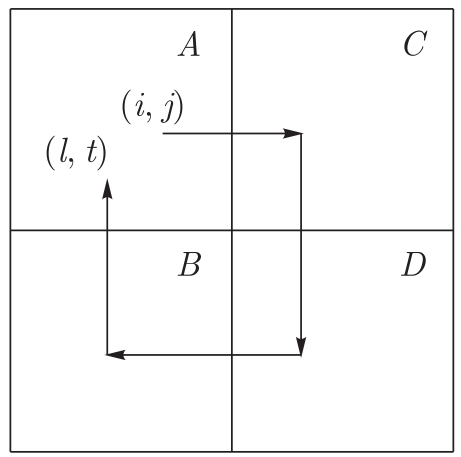

Рис. 3

Определение. а) Пусть $A, B$ - два ЛК порядка $n$ на множестве $\{0, \ldots, n-1\}$ и $T_{A}, T_{B}$ - трафареты (т. е. $(0,1)$-матрицы) порядка $n$. Трафареты $T_{A}, T_{B}$ называются строчно согласованныли, если при действии подстановки $\sigma_{A B}$ трафарет $T_{A}$ переходит в инвертированный по отношению к $T_{B}$ трафарет $\overline{T_{B}}\left(\overline{T_{B}}\right.$ получается из $T_{B}$ заменой 0 на 1 и наоборот). Аналогично с помощью подстановки $\kappa_{A B}$ определяется столбцовая согласованность трафаретов.

б) Пусть $A, B, C, D-4$ ЛК порядка $n$ на множестве $\{0, \ldots, n-1\}$ и $T_{A}, T_{B}, T_{C}, T_{D}-4$ трафарета порядка $n$. Будем говорить, что трафареты $T_{A}, T_{B}, T_{C}, T_{D}$ согласованы с набором ЛК $A, B, C, D$, если строчно согласованы $T_{A}, T_{C}$ и $T_{B}, T_{D}$ и столбцово согласованы $T_{A}, T_{B}$ и $T_{C}, T_{D}$.

Из этих определений и свойства подстановки $\chi_{A C D B}$, сформулированного в замечании, очевидным образом вытекает следующее утверждение.

Утверждение 1. Набор четырех ЛК и четырех трафаретов $\left(A, B, C, D, T_{A}, T_{B}, T_{C}, T_{D}\right)$ задает распавшийся латинский квадрат порядка $2 n$ на множестве $\{0, \ldots, 2 n-1\}$ в том и только том случае, когда трафареты $T_{A}, T_{B}, T_{C}, T_{D}$ согласованы с набором ЛК $A, B, C, D$. При этом:

a) трафареть $T_{B}, T_{C}, T_{D}$ получаются из $T_{A}$ с помощью строчных и столбиовых подстановок и инвертирования;

б) исходный трафарет $T_{A}$ задает таким способом согласованный набор трафаретов $T_{A}, T_{B}, T_{C}, T_{D}$ в том и только том случае, когда $T_{A}$ постоянен на ииклах подстановки $\chi_{A C D B}$;

в) существует ровно $2^{k}$ различных трафаретов $T_{A}$, порождающих согласованный набор трафаретов $T_{A}, T_{B}, T_{C}, T_{D}$, где $k$ - число ииклов в подстановке $\chi_{A C D B}$. 
ЗАМЕЧАНИЕ. Далее будем использовать следующее обозначение для распавшегося ЛК $F$ порядка $2 n: F=\left(A, B, C, D, T_{A}\right)$, где $A, B, C, D$ - латинские квадраты порядка $n$ на множестве $\{0, \ldots, n-1\}$ и $T_{A}-$ трафарет, удовлетворяющий п. б) утверждения 1. Такой трафарет будем называть $c o$ гласованным с набором $A, B, C, D$. Если $F$ является однородным ЛК, т. е. $A=B=C=D$, то его будем обозначать так: $F=\left(A, T_{A}\right)$.

Итак, по любому заданному набору $A, B, C, D$ ЛК порядка $n$ на множестве $\{0, \ldots, n-1\}$ можно построить ровно $2^{k}$ различных распавшихся ЛК $F$ порядка $2 n$ на множестве $\{0, \ldots, 2 n-1\}$, где $k$ - число циклов в подстановке $\chi_{A C D B}, n \leq k \leq n^{2}$.

Далее наряду с общим случаем распавшегося ЛК $F$ будут рассматриваться два частных случая:

1) Распавшийся ЛК $F$ имеет тождественную подстановку $\chi_{A C D B}$.

2) Распавшийся ЛК является однородным: $F=\left(A, T_{A}\right)$; тогда, очевидно, подстановка $\chi_{A C D B}$ тождественна.

В указанных частных случаях любой трафарет $T_{A}$ является согласованным и число различных распавшихся ЛК рассматриваемого вида максимально и равно $2^{n^{2}}$ (при фиксированном наборе четырех исходных ЛК порядка $n)$.

Следующее утверждение непосредственно вытекает из утверждения 1.

Утверждение 2. Пусть $F=\left(A, B, C, D, T_{A}\right)-$ распавшийся ЛК порядка 2n. Пусть $d_{1}, \ldots, d_{n}$ - диагонали ЛК А. Тогда для любого $i \in\{1, \ldots, n\}$ трафарет $T_{A}^{(i)}$, получаюшийся из $T_{A}$ инвертированием элементов, находящихся на диагонали $d_{i}$, является согласованным с набором ЛК $A, B, C, D$, и соответствуюший ему распавшийся ЛК $F^{(i)}=\left(A, B, C, D, T_{A}^{(i)}\right)$ главноизотопен исходному ЛК $F$.

Следствие. Пусть $\mathcal{M}=\left\{\left(A, B, C, D, T_{A}\right) \mid T_{A}\right.$ согласован с $\left.A, B, C, D\right\}-$ множество всех распавшихся ЛК, задаваемых четверкой $A, B, C, D$, $|\mathcal{M}|=2^{k}$. Тогда $\mathcal{M}$ распадается на $2^{k-n}$ не пересекаюшихся между собой подмножеств мощзности $2^{n}$ каждое, состоящих из главноизотопных между собой распавшихся ЛК. В частности, у любых двух ЛК из одного такого подмножества множества их трансверсалей совпадают.

ЗАМЕчАНИЯ. 1) Если число $k$ циклов подстановки $\chi_{A C D B}$ равно $n$, то все $2^{n}$ распавшихся ЛК порядка $2 n$, соответствующих такому набору $A, B, C, D$, главноизотопны между собой и изотопны ЛК, определяемому трафаретом $T_{A}=(1)_{n \times n}$. Нетрудно видеть, что при нечетном $n$ распавшийся ЛК с таким трафаретом вовсе не имеет трансверсалей. 
2) Если число $k$ циклов подстановки $\chi_{A C D B}$ равно $n+1$, то имеем 2 класса главноизотопных ЛК. В самом деле, если $d=c_{1} \cup c_{2}$ - диагональ ЛК $A$, распадающаяся на 2 цикла подстановки $\chi_{A C D B}$, то все распавшиеся ЛК $F=\left(A, B, C, D, T_{A}\right)$, у которых значения $T_{A}$ на циклах $c_{1}$ и $c_{2}$ одинаковы, главноизотопны распавшемуся ЛК с трафаретом $T_{A}=(1)_{n \times n}$. При нечетном $n$ такие ЛК трансверсалей не имеют. Второй класс главноизотопных ЛК определяется трафаретами $T_{A}$, у которых значения на $c_{1}$ и $c_{2}$ не совпадают.

3) В приложении приведены примеры (построенных с помощью вычислительной техники) наборов ЛК $A, B, C, D$ порядка 5 , порождающие подстановки $\chi_{A C D B}$ с любым наперед заданным числом циклов $k \in\{5, \ldots, 25\}$.

\section{3. Проекции трансверсалей распавшихся ЛК}

Пусть $F=\left(A, B, C, D, T_{A}\right)$ - распавшийся ЛК порядка $2 n$ на множестве $\{0, \ldots, 2 n-1\}$, определяемый четверкой $A, B, C, D$ латинских квадратов порядка $n$ на множестве $\{0, \ldots, n-1\}$ и согласованным с ней трафаретом $T_{A}$.

Будем использовать обозначение $G=F \bmod n$ для результата приведения элементов $F$ по модулю $n$.

Определение. Проекцией $\pi_{A}:\{1, \ldots, 2 n\}^{2} \rightarrow\{1, \ldots, n\}^{2}$ позиций $(i, j) \in\{1, \ldots, 2 n\}^{2}$ латинского квадрата $F$ на позиции латинского квадрата $A$ называется отображение

$$
\pi_{A}(i, j)=\left\{\begin{array}{lll}
(i, j) & \text { при } & (i, j) \in\{1, \ldots, n\}^{2}, \\
(l, t) & \text { при } & (i, j) \notin\{1, \ldots, n\}^{2},
\end{array}\right.
$$

где во втором случае $(l, t)$ получается из $(i, j) \notin\{1, \ldots, n\}^{2}$ последовательным применением строчных и (или) столбцовых подстановок по часовой стрелке до первого попадания в ЛК $A$ по схеме, изображенной на рисунке 4.

Пусть $\gamma \subseteq\{1, \ldots, 2 n\}^{2}-$ некоторое множество позиций ЛК $F$. Проекцию $\underline{\lambda}=\pi_{A}(\gamma)$ будем рассматривать как базовое множество для мультимножества $\lambda=\left(\underline{\lambda}, \Pi_{\lambda}\right)$, задаваемого с помощью целочисленной матрицы $\Pi_{\lambda}=\left(\pi_{i j}\right)_{n \times n}$ порядка $n$, в которой элемент $\pi_{i j}$ равен числу различных позиций из множества $\gamma$, проецирующихся на позицию $(i, j) \in\{1, \ldots, n\}^{2}$ :

$$
\pi_{i j}=\left|\left\{m \in \gamma \mid \pi_{A}(m)=(i, j)\right\}\right|
$$


Мультимножество $\lambda$ содержит элемент $(i, j)$ с кратностью $\pi_{i j}$. В описанной ситуации будем говорить, что мультимножество $\lambda$ является проекцией множества $\gamma$, и обозначать $\lambda=$ $=\pi_{A}(\gamma)$. При этом $\lambda$ будет обычным множеством тогда и только тогда, когда $\Pi_{\lambda}$ является $(0,1)$-матрицей.

Определение. Пусть заданы ЛК $U$ порядка $m$ на $\{0, \ldots, m-1\}$ и некоторое мультимножество $\lambda, \underline{\lambda} \subseteq\{1, \ldots, m\}^{2}$ его позиций. Будем говорить, что $\lambda$ является $k$-сбалан-

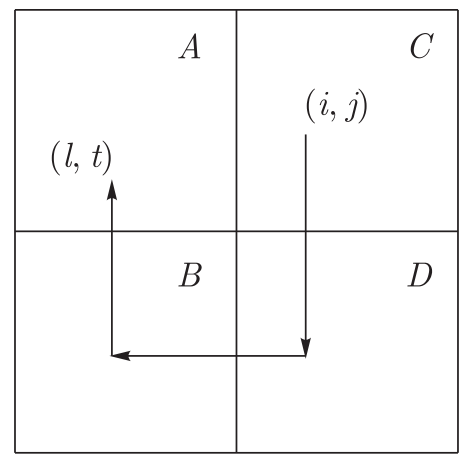

Рис. 4 сированным, $k \in\{1, \ldots, m\}$, если каждый элемент $a \in\{0, \ldots, m-1\}$ встречается на позициях этого мультимножества ровно $k$ раз с учетом кратности вхождения позиций в $\lambda$.

Следующее утверждение характеризует мультимножества, получающиеся при проецировании трансверсалей распавшегося ЛК $F$.

Утверждение 3. Пусть $\tau \subset\{1, \ldots, 2 n\}^{2}-$ трансверсаль ЛК $F=$ $=\left(A, B, C, D, T_{A}\right)$. Тогда ее проекиия $\pi_{A}(\tau)$ является 2-сбалансированным мультимножеством позищий ЛК $А$, удовлетворяющим следующим дополнительным условиям:

1) Любой элемент $(i, j) \in \pi_{A}(\tau)$, принадлежащий циклу длины 1 подстановки $\chi_{A C D B}$, имеет в $\pi_{A}(\tau)$ кратность 1.

2) Элемент $(i, j) \in \pi_{A}(\tau)$ кратности больше 1 имеет кратность 2. В этом случае $(i, j)$ принадлежит ииклу длины $r \geq 2$ подстановки $\chi_{A C D B}$, и в него проецируются 2 элемента трансверсали $\tau:$ сам $(i, j) \in \tau$ и элемент $(l, t)$ из подматрищы $C$ матрищь $G$, как показано на рисунке 5 .

3) Два элемента $\left(i_{1}, j_{1}\right),\left(i_{2}, j_{2}\right) \in \pi_{A}(\tau)$ кратностей 1 каждый и лежашие на одном ичикле подстановки $\chi_{A C D B}$, являются результатами проецирования элементов $\left(l_{1}, t_{1}\right),\left(l_{2}, t_{2}\right) \in \tau$, лежаших в смежных латинских квадратах матриць $G$.

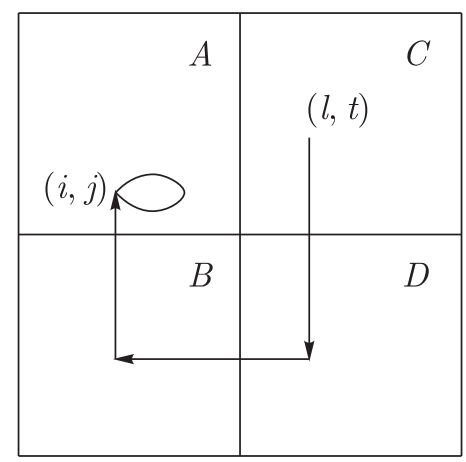

Puc. 5

Доказательство. Мультимножество $\pi_{A}(\tau)$ позиций ЛК $A$ является 2-сбалансированным, так как на позициях трансверсали $\tau$ в ЛК $F$ каждое 
число $a \in\{0, \ldots, 2 n-1\}$ встречается по одному разу, и поэтому на позициях $\pi_{A}(\tau)$ в ЛК $A$ каждое число $b \in\{0, \ldots, n-1\}$ встречается по два раза (с учетом кратности позиций). Справедливость свойства 1) легко видна из приведенной на рисунке 6 схемы, показывающей, как образуется цикл длины 1 подстановки $\chi_{A C D B}$.

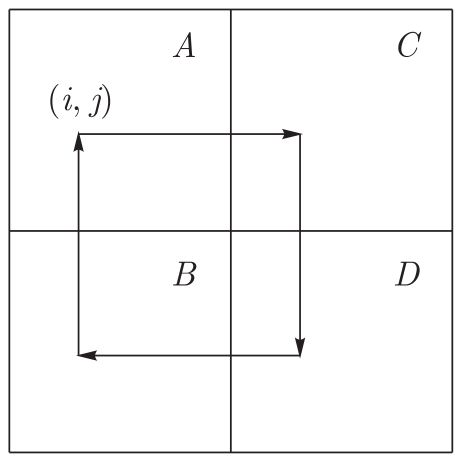

Рис. 6

Покажем, что если $\tau$ является трансверсалью в $F$, то элемент $(i, j) \in \pi_{A}(\tau)$ не может иметь кратность больше 1 . Действительно, два различных элемента $\tau$ не могут проецироваться в $(i, j)$, поскольку если они находятся в смежных квадратах, то это противоречит тому, что $\tau$ является подстановкой, а если они находятся в несмежных квадратах, то на их местах в ЛК $F$ стоят одинаковые элементы и $\tau-$ не трансверсаль.

П. 2) также вытекает из того, что $\tau$ является трансверсалью в $F$. Действительно, $\pi_{A}(\tau)$ не может иметь элементов кратности больше 2.

Рассмотрение схемы, приведенной в формулировке этого пункта, показывает, что на ней изображен единственный вариант, не противоречащий тому, что $\tau-$ трансверсаль.

Для доказательства п. 3) заметим, что так как $\left(i_{1}, j_{1}\right),\left(i_{2}, j_{2}\right) \in \pi_{A}(\tau)$ лежат на одном цикле подстановки $\chi_{A C D B}$, то они принадлежат одной и той же диагонали ЛК $A$, т. е. $a_{i_{1} j_{1}}=a_{i_{2} j_{2}}$. Если бы позиции $\left(l_{1}, t_{1}\right),\left(l_{2}, t_{2}\right) \in \tau$, проецирующиеся на $\left(i_{1}, j_{1}\right),\left(i_{2}, j_{2}\right) \in \pi_{A}(\tau)$, лежали бы в несмежных подматрицах матрицы $G$, то по построению распавшегося ЛК $F$ имело бы место равенство двух элементов трансверсали $\tau: f_{l_{1} t_{1}}=f_{l_{2} t_{2}}$.

Следствие. 1) Если подстановка $\chi_{A C D B}$ тождественна $u \tau \in \operatorname{Tr}(F)$, то $\pi_{A}(\tau)$ является 2-сбалансированным множеством позиций ЛК $А$.

2) Если подстановка $\chi_{A C D B}$ тождественна и трансверсаль $\tau$ такова, что множество $\left(\kappa_{B A}, \kappa_{D C}\right)(\tau \cap(\{n+1, \ldots, 2 n\} \times\{1, \ldots, 2 n\}))$ представляет все строки, в $\{1, \ldots, n\} \times\{1, \ldots, 2 n\}, a\left(\sigma_{C A}, \sigma_{D B}\right)(\tau \cap(\{1, \ldots, 2 n\} \times$ $\times\{n+1, \ldots, 2 n\}))-$ все столбиы в $\{1, \ldots, 2 n\} \times\{1, \ldots, n\}$, то $\pi_{A}(\tau)$ является 2-трансверсалью ЛК А.

3) $Е с л и ~ F=\left(A, T_{A}\right)$ - однородный ЛК и $\tau \in \operatorname{Tr}(F)$, то $\pi_{A}(\tau)$ является 2-трансверсалью ЛК А. 
Доказательство. 1) Поскольку на трансверсали $\tau$ в ЛК $F$ каждый элемент из $\{0, \ldots, 2 n-1\}$ встречается ровно один раз, то в проекции $\pi_{A}(\tau)$ каждый элемент из $\{0, \ldots, n-1\}$ встретится ровно два раза.

2) Действительно, в этом случае $\pi_{A}(\tau)$ - 2-сбалансированное множество позиций ЛК $A$, которое содержит ровно по две позиции из каждого столбца и каждой строки этого латинского квадрата, т. е. является его 2-трансверсалью.

3) Очевидным образом вытекает из пункта 2).

Определение. Назовем 2-сбалансированное мультимножество $\lambda, \underline{\lambda} \subset$ $\subset\{1, \ldots, n\}^{2}$, подстановочным 2-сбалансированным мультимножеством позиций ЛК $A$ (для заданного набора ЛК $A, B, C, D$ ), порожденным подстановкой $\alpha \subset\{1, \ldots, 2 n\}^{2}$ степени $2 n$, если $\pi_{A}(\alpha)=\lambda$ и пара $(\lambda, \alpha)$ обладает свойствами 1)-3) из утверждения 3. Если при этом $\lambda$ является множеством, то будем говорить о подстановочном 2-сбалансированном множестве, порожденном подстановкой $\alpha \subset\{1, \ldots, 2 n\}^{2}$.

ЗАМЕЧАНИЯ. 1) Если $F$ - распавшийся ЛК с тождественной подстановкой $\chi_{A C D B}$, то этому определению удовлетворяют все 2-сбалансированные множества позиций ЛК $A$, являющиеся проекциями подстановок степени $2 n$.

2) Легко видеть, что в случае однородного ЛК $F=\left(A, T_{A}\right)$ 2-сбалансированными подстановочными множествами могут быть только 2-трансверсали ЛК $A$, поскольку если проекция $\pi_{A}(\alpha)=\lambda$ подстановки $\alpha$ на ЛК $A$ является множеством, то $\lambda$ имеет ровно по 2 элемента в каждой строке и в каждом столбце ЛК $A$; а поскольку $\lambda$ является 2-сбалансированным, то оно - 2-трансверсаль в $A$.

Следующее утверждение показывает, что верно и обратное: в однородном случае любая 2-трансверсаль ЛК $A$ является 2-сбалансированным подстановочным множеством.

Утверждение 4. Пусть $F=\left(A, T_{A}\right)-$ однородный ЛК порядка $2 n$ и $\lambda$ - произвольная 2-трансверсаль ЛК А. Тогда существует ровно $2^{2 n}$ подстановок $\alpha \subset\{1, \ldots, 2 n\}^{2}$ степени $2 n$, проеиирующихся в $\lambda$ : $\left|\left\{\alpha \in S_{2 n} \mid \pi_{A}(\alpha)=\lambda\right\}\right|=2^{2 n}$.

Доказательство. Рассмотрим $(0,1)$-матрицу $S=\left(s_{i j}\right)_{n \times n}$ порядка $n$, в которой $s_{i j}=1 \Leftrightarrow(i, j) \in \lambda$; матрица $S$ соответствует 2-трансверсали $\lambda$ ЛК $A$. В этой матрице в каждой строке и каждом столбце ровно по две единицы, поэтому $S=\Pi_{1}+\Pi_{2}-$ сумма двух подстановочных матриц. Рассмотрим $(0,1)$-матрицу порядка $2 n$, в которой в первых $n$ строках и столбцах стоит подматрица, равная $S$, а остальные элементы равны 0 (см. рис. 7): 


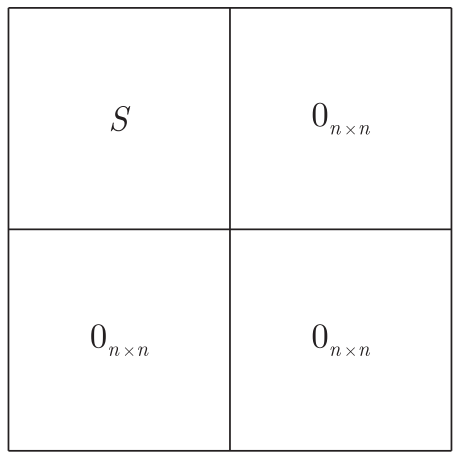

Рис. 7

Теперь для доказательства утверждения необходимо перераспределить единицы из $S$ на одноименные места в четырех подматрицах порядка $n$ так, чтобы в итоге получилась подстановочная матрица. Единицы, входящие в $\Pi_{1}$, распределяем по подматрицам на одноименные места произвольным образом. Всего получается $4^{n}=2^{2 n}$ вариантов. Очевидно, что для каждого такого варианта существует единственный вариант распределения единиц из подстановки $\Pi_{2}$, в результате которого получается подстановочная матрица порядка $2 n$.

Пусть $\lambda\left(\underline{\lambda} \subset\{1, \ldots, n\}^{2}\right)$ - 2-сбалансированное мультимножество, порождаемое подстановкой $\alpha \subset\{1, \ldots, 2 n\}^{2}$ степени $2 n: \pi_{A}(\alpha)=\lambda$. Будем изучать множество $\operatorname{Tr}(F, \lambda)$ всех трансверсалей $\tau$ латинского квадрата $F=\left(A, B, C, D, T_{A}\right)$, порождающих $\lambda$ :

$$
\operatorname{Tr}(F, \lambda)=\left\{\tau \in \operatorname{Tr}(F) \mid \pi_{A}(\tau)=\lambda\right\} .
$$

Пусть $C_{1}, \ldots C_{k} \subset\{1, \ldots, n\}^{2}-$ все циклы подстановки $\chi_{A C D B}$ и $\eta=\bigcup_{\substack{\nu \in\{1, \ldots, k\}: \\ C_{\nu} \cap \lambda \neq \varnothing}} C_{\nu}-$ объединение тех и только тех циклов, которые имеют общие элементы с $\lambda$.

Через $H=\left(h_{i j}\right)_{n \times n}$ обозначим $(0,1)$-матрицу порядка $n$, соответствующую $\eta$ :

$$
h_{i j}=1 \Leftrightarrow(i, j) \in \eta \text {. }
$$

Рассмотрим новый трафарет $T_{A}^{\prime}=T_{A} * H$. Тогда очевидно, $T_{A}^{\prime}$ согласован с четверкой ЛК $A, B, C, D$, и они совместно задают новый распавшийся ЛК $F^{\prime}=\left(A, B, C, D, T_{A}^{\prime}\right)$. Очевидно также, что множества трансверсалей латинских квадратов $F$ и $F^{\prime}$, проецирующихся на $\lambda$, совпадают: $\operatorname{Tr}(F, \lambda)=\operatorname{Tr}\left(F^{\prime}, \lambda\right)$. Поэтому при изучении множества $\operatorname{Tr}(F, \lambda)$ для произвольных распавшихся ЛК $F$ полезно следующее понятие.

Определение. 1) Пусть $\lambda$ - произвольное мультимножество позиций ЛК $A$ и $T_{A}^{\prime} \in\{0,1\}^{n \times n}-$ произвольный трафарет. Трафарет $T_{A}^{\prime}$ называется накрывающим мультимножество $\lambda$, если его элементы $t_{i j}^{\prime}$ удовлетворяют 
условию

$$
t_{i j}^{\prime}=0 \quad \forall(i, j) \in\{1, \ldots, n\}^{2} \backslash \eta, \quad \text { где } \quad \eta=\bigcup_{\substack{\nu \in\{1, \ldots, k\}: \\ C_{\nu} \cap \lambda \neq \varnothing}} C_{\nu} .
$$

2) Трафарет $T_{A}^{\prime}$, накрывающий мультимножество $\lambda$ и построенный по трафарету $T_{A}$ в рассуждении перед данным определением, будем называть трафаретом, накрывающим мультимножество $\lambda$ и соответствующим исходному трафарету $T_{A}$.

ЗАмЕчАНИЯ. 1) При изучении множеств $\operatorname{Tr}(F, \lambda)$ для всевозможных распавшихся ЛК $F=\left(A, B, C, D, T_{A}\right)$ достаточно ограничиться рассмотрением только трафаретов $T_{A}$, накрывающих $\lambda$.

2) Если подстановка $\chi_{A C D B}$ тождественна, то накрывающим для $\lambda$ будет любой трафарет, в котором на позициях $(i, j) \notin \lambda$ стоят нули.

Рассмотрим теперь следующую задачу. Пусть для заданной четверки ЛК $A, B, C, D$ порядка $n$ задано также подстановочное 2-сбалансированное мультимножество позиций $\lambda$ ЛК $A$ вместе с любой из порождающих его подстановок $\alpha \subset\{1, \ldots, 2 n\}^{2}$ степени $2 n: \pi_{A}(\alpha)=\lambda$. Можно ли так подобрать трафарет $T_{A}$, согласованный с набором $A, B, C, D$, чтобы подстановка $\alpha$ была трансверсалью в ЛК $F=\left(A, B, C, D, T_{A}\right)$, т. е. $\alpha \in \operatorname{Tr}(F)$ ? Если да, то сколько таких трафаретов существует? Как было отмечено выше, достаточно ограничиться рассмотрением только трафаретов $T_{A}$, накрывающих $\lambda$.

В следующем определении вводятся наборы, являющиеся как бы маркерами того, что $\alpha \in \operatorname{Tr}(F)$.

Определение. Пусть $F=\left(A, B, C, D, T_{A}\right)-$ распавшийся ЛК, $G=$ $=F \bmod n=\left(g_{i j}\right)_{2 n \times 2 n}=\left(\begin{array}{ll}A & C \\ B & D\end{array}\right)_{2 n \times 2 n}-$ результат приведения элементов $F$ по модулю $n, \alpha \subset\{1, \ldots, 2 n\}^{2}-$ подстановка степени $2 n$. Рассмотрим следующие упорядоченные наборы:

$$
\begin{aligned}
L_{1}= & \left(i_{1}, \ldots, i_{r}\right): f_{i_{s} \alpha\left(i_{s}\right)}=g_{i_{s} \alpha\left(i_{s}\right)} \in\{0, \ldots, n-1\}, s \in\{1, \ldots, r\}, \\
L_{2}= & \left(j_{1}, \ldots, j_{2 n-r}\right): f_{j_{s} \alpha\left(j_{s}\right)}=g_{j_{s} \alpha\left(j_{s}\right)}+n \in\{n, \ldots, 2 n-1\}, s \in\{1, \ldots, 2 n-r\}, \\
M_{1}= & \left(m_{1}, \ldots, m_{r}\right): m_{s}=f_{i_{s} \alpha\left(i_{s}\right)}=g_{i_{s} \alpha\left(i_{s}\right)} \in\{0, \ldots, n-1\}, s \in\{1, \ldots, r\}, \\
M_{2}= & \left(\bar{m}_{1}, \ldots, \bar{m}_{2 n-r}\right): \\
& \bar{m}_{s}=g_{j_{s} \alpha\left(j_{s}\right)}=r_{n}\left(f_{j_{s} \alpha\left(j_{s}\right)}\right) \in\{0, \ldots, n-1\}, s \in\{1, \ldots, 2 n-r\} .
\end{aligned}
$$

Здесь $r_{n}$ означает взятие остатка от деления на $n$.

Через $\widetilde{M}_{1}$ и $\widetilde{M}_{2}$ обозначим множества элементов, встречающихся в наборах $M_{1}$ и $M_{2}$ соответственно. 
ЗАМЕЧАНИЯ. 1) Подстановка $\alpha$ является трансверсалью в ЛК $F$ тогда и только тогда, когда $\widetilde{M}_{1}=\widetilde{M}_{2}=\{0, \ldots, n-1\}$.

2) Для того, чтобы $\alpha$ была трансверсалью в $F$, необходимо, чтобы $r=n$.

Следующая лемма показывает, каким образом изменяются наборы $L_{1}, L_{2}, M_{1}, M_{2}$ при изменении значений трафарета $T_{A}$ на местах, соответствующих одному произвольному циклу $C_{\nu}$ подстановки $\chi_{A C D B}$.

Лемма 1. Пусть $\lambda$ - подстановочное 2-сбалансированное мультимножество позиций ЛК $A$, порожденное подстановкой $\alpha$ степени $2 n$, для которой построены указанные в предыдущем определении наборы $L_{1}, L_{2}, M_{1}, M_{2}$.

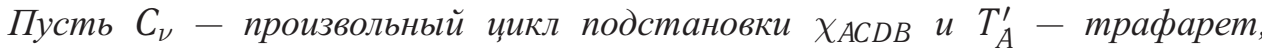
полученный из $T_{A}$ изменением значений его элементов на позициях ияикла $C_{\nu}$ на противоположные. Пусть $F^{\prime}=\left(A, B, C, D, T_{A}^{\prime}\right)-$ соответствуюший распавшийся ЛК и $L_{1}^{\prime}, L_{2}^{\prime}, M_{1}^{\prime}, M_{2}^{\prime}$ - новые наборы из определения, соответствуюшие подстановке $\alpha$. Тогда:

1) Если $C_{\nu} \cap \lambda=\varnothing$, то $\left(L_{1}, L_{2}, M_{1}, M_{2}\right)=\left(L_{1}^{\prime}, L_{2}^{\prime}, M^{\prime}{ }_{1}, M^{\prime}{ }_{2}\right)$.

2) Пусть $C_{\nu} \cap \lambda \neq \varnothing$. Тогда возможны следующие варианты:

2.1. $C_{\nu} \cap \lambda=\{p\}$, где $p$ - элемент кратности $1, p=(l, s) \in\{1, \ldots, n\}^{2}$. Тогда при переходе от $F \kappa F^{\prime}$ происходит переход одного элемента из наборов с номером 1 в наборы с номером 2 или наоборот.

2.2. $C_{\nu} \cap \lambda=\{p\}$, где $p$ - элемент кратности 2. Тогда при переходе от $F \kappa F^{\prime}$ происходит переход одного элемента из наборов с номером 1 в наборы с номером 2 и переход одного элемента из наборов с номером 2 в наборы с номером 1. При этом наборы $M_{1}$ и $M_{2}$ обмениваются одинаковыми элементами, т.е. $\widetilde{M}_{1}=\widetilde{M}_{1}^{\prime}, \widetilde{M}_{2}=\widetilde{M}_{2}^{\prime}$.

2.3. $C_{\nu} \cap \lambda=\left\{p_{1}, p_{2}\right\}, p_{1} \neq p_{2}$ - элементы $\lambda$ кратности 1. В этом случае при переходе от $F \kappa F^{\prime}$ происходит такой же обмен элементами между наборами с номерами 1 и 2, как и в случае 2.2.

Доказательство. 1) Очевидно, так как в этом случае значения трафаретов $T_{A}$ и $T_{A}^{\prime}$ на позициях, входящих в $\lambda$, одинаковы.

2) Этот пункт распадается на три подпункта, поскольку цикл является подмножеством некоторой диагонали ЛК $A$.

2.1. Пусть $(i, \alpha(i)) \in \alpha-$ единственный элемент подстановки $\alpha$, проецирующийся в $p$. Если в ЛК $F=\left(f_{i j}\right)_{2 n \times 2 n}$ для элемента $f_{i \alpha(i)}$ справедливо включение $f_{i \alpha(i)} \in\{0, \ldots, n-1\}$, то в ЛК $F^{\prime}=\left(f_{i j}^{\prime}\right)_{2 n \times 2 n}$ элемент $f_{i \alpha(i)}^{\prime} \in\{n, \ldots, 2 n-1\}$ и наоборот, т. е. происходит указанный в этом пункте леммы переход элементов. 
2.2. Пусть в элемент $p \in \lambda$ кратности 2 проецируются 2 элемента из подстановки $\alpha:\left(i_{1}, \alpha\left(i_{1}\right)\right),\left(i_{2}, \alpha\left(i_{2}\right)\right), i_{1} \neq i_{2}$, причем в соответствии с п. 2) утверждения 3 выполнены включения $i_{1}, i_{2} \in\{1, \ldots, n\}$. Тогда по построению распавшегося ЛК $F$ его элементы $f_{i_{1} \alpha\left(i_{1}\right)}, f_{i_{2} \alpha\left(i_{2}\right)}$, лежащие в двух смежных верхних подматрицах, удовлетворяют соотношениям $f_{i_{1} \alpha\left(i_{1}\right)} \equiv f_{i_{2} \alpha\left(i_{2}\right)} \bmod n, f_{i_{1} \alpha\left(i_{1}\right)} \neq f_{i_{2} \alpha\left(i_{2}\right)}$. Поэтому при переходе от $F$ к $F^{\prime}$ происходит переход элементов между наборами, указанный в п. 2.2 леммы.

2.3. В данном случае согласно определению порождающей подстановки, подстановка $\alpha$ удовлетворяет п. 3) утверждения 3 , а ее элементы $\left(i_{1}, j_{1}\right),\left(i_{2}, j_{2}\right)$, проецирующиеся в $p_{1}$ и $p_{2}$, лежат в смежных подматрицах матрицы $G$ и, следовательно, $f_{i_{1} \alpha\left(i_{1}\right)} \equiv f_{i_{2} \alpha\left(i_{2}\right)} \bmod n, f_{i_{1} \alpha\left(i_{1}\right)} \neq f_{i_{2} \alpha\left(i_{2}\right)}$, т. е. при переходе от $F$ к $F^{\prime}$ происходит переход элементов между наборами такой же, как и в п. 2.2 леммы.

ЗАМЕЧАНИЕ. Если $F$ - распавшийся ЛК с тождественной подстановкой $\chi_{A C D B}$, то в п. 2) леммы возможен только подпункт 2.1 .

Утверждение 5. Пусть $A, B, C, D-$ - абор ЛК порядка $n, \lambda-$ подстановочное 2-сбалансированное мультимножество позиций ЛК А и $\alpha-$ порождающая его подстановка степени $2 n$. Тогда существует ровно $2^{n}$ таких согласованных с $A, B, C, D$ и накрывающих $\lambda$ трафаретов $T_{A}$, что для латинского квадрата $F=\left(A, B, C, D, T_{A}\right)$ подстановка $\alpha$ является трансверсалью: $\alpha \in \operatorname{Tr}(F, \lambda)$.

Доказательство. 1) Сначала построим один трафарет $T_{A}$, обладающий указанным в утверждении свойством. Пусть $T_{A}=(0)_{n \times n}$. Если $\alpha \in \operatorname{Tr}(F)$, то этот трафарет - искомый. Если $\alpha \notin \operatorname{Tr}(F)$, то $\widetilde{M}_{1} \neq \widetilde{M}_{2}$ для подстановки $\alpha$. В 2-сбалансированном мультимножестве $\lambda$ позиций ЛК $A$ переберем пары элементов, на местах которых в $A$ стоят одинаковые значения (при этом элемент кратности 2 считается «парой элементов»). Для любой такой пары возможны следующие варианты:

a) Если $p \in \lambda$ - элемент кратности 2, то, согласно определению подстановочного 2-сбалансированного мультимножества, для него выполнен п. 2) утверждения 3, т. е. в него проецируются 2 элемента из $\alpha:\left(i_{1}, \alpha\left(i_{1}\right)\right)=p$ и $\left(i_{2}, \alpha\left(i_{2}\right)\right): i_{2} \in\{1, \ldots, n\}, \alpha\left(i_{2}\right) \in\{n+1, \ldots, 2 n\}$. Тогда $f_{i_{1} \alpha\left(i_{1}\right)} \equiv$ $\equiv f_{i_{2} \alpha\left(i_{2}\right)} \bmod n, f_{i_{1} \alpha\left(i_{1}\right)} \neq f_{i_{2} \alpha\left(i_{2}\right)}$, т. е. либо $i_{1} \in L_{1} \wedge i_{2} \in L_{2}$, либо $i_{2} \in L_{1} \wedge i_{1} \in$ $\in L_{2}$; это означает, что элементу $p \in \lambda$ соответствуют два равных элемента, один из которых лежит в наборе $M_{1}$, а другой - в $M_{2}$.

б) Пусть $p_{1}, p_{2} \in \lambda-$ элементы кратности 1 , лежащие на одном цикле подстановки $\chi_{A C D B}$; стоящие на этих местах элементы ЛК $A$ равны 
между собой: $a_{p_{1}}=a_{p_{2}}$. Тогда, согласно определению подстановочного 2сбалансированного мультимножества, для $p_{1}, p_{2}$ выполнен п. 3) утверждения 3 , т. е. $p_{1}, p_{2}$ являются результатами проецирования элементов $\left(i_{1}, \alpha\left(i_{1}\right)\right)$ и $\left(i_{2}, \alpha\left(i_{2}\right)\right)$ подстановки $\alpha$, лежащих в смежных подматрицах матрицы $G$. Тогда, как и в предыдущем случае, $f_{i_{1} \alpha\left(i_{1}\right)} \equiv f_{i_{2} \alpha\left(i_{2}\right)} \bmod n, f_{i_{1} \alpha\left(i_{1}\right)} \neq f_{i_{2} \alpha\left(i_{2}\right)}$, т. е. либо $i_{1} \in L_{1} \wedge i_{2} \in L_{2}$, либо $i_{2} \in L_{1} \wedge i_{1} \in L_{2}$. Это означает, что элементам $p_{1}, p_{2} \in \lambda$ соответствуют два равных элемента, один из которых лежит в наборе $M_{1}$, а другой - в $M_{2}$.

в) Если бы перебор пар элементов из $\lambda$ с одинаковым заполнением в ЛК $A$ исчерпывался только предыдущими двумя случаями, то $\alpha$ была бы трансверсалью в $F$. По предположению это не так, значит существуют элементы $p_{1}, p_{2} \in \lambda$ кратности 1 , принадлежащие различным циклам подстановки $\chi_{A C D B}$ и удовлетворяющие условию $a_{p_{1}}=a_{p_{2}}$. Пусть $\pi_{A}\left(i_{1}, \alpha\left(i_{1}\right)\right)=p_{1}$ и $\pi_{A}\left(i_{2}, \alpha\left(i_{2}\right)\right)=p_{2}$. Тогда $a_{p_{1}}=a_{p_{2}} \equiv f_{i_{1} \alpha\left(i_{1}\right)} \equiv f_{i_{2} \alpha\left(i_{2}\right)} \bmod n$. Если $i_{1}$ и $i_{2}$ лежат в различных наборах $L_{1}$ и $L_{2}$, то и в $M_{1}$, и в $M_{2}$ входит элемент $h=$ $a_{p_{1}}=a_{p_{2}}$. Если $i_{1}$ и $i_{2}$ лежат в одном и том же наборе, например в $L_{1}$, то в $M_{1}$ имеется два одинаковых элемента, равных $h=a_{p_{1}}=a_{p_{2}}$. В этом случае воспользуемся п. 2.1 леммы 1 и изменим значения трафарета $T_{A}$ на местах цикла подстановки $\chi_{A C D B}$, которому принадлежит $p_{1}$, на противоположные. Тогда один из элементов, равных $h=a_{p_{1}}=a_{p_{2}}$, покинет набор $M_{1}$ и перейдет в $M_{2}$.

После завершения описанного перебора получим накрывающий $\lambda$ трафарет $T_{A}^{\prime}$, задающий распавшийся ЛК $F^{\prime}=\left(A, B, C, D, T^{\prime}{ }_{A}\right)$, для которого $\alpha \in \operatorname{Tr}\left(F^{\prime}, \lambda\right)$.

2) Теперь в нашем распоряжении имеется по крайней мере один накрывающий $\lambda$ трафарет $T_{A}$, для которого $\alpha \in \operatorname{Tr}(F, \lambda), F=\left(A, B, C, D, T_{A}\right)$.

Рассмотрим $h \in\{0, \ldots, n-1\}$ и места $p_{1}, p_{2} \in \lambda$ с условием $h=a_{p_{1}}=a_{p_{2}}$.

Если $p_{1}=p_{2}=p-$ элемент кратности 2 , лежащий на цикле $C_{\nu}$ подстановки $\chi_{A B C D}$, или $p_{1} \neq p_{2}-$ элементы кратности 1 , лежащие на одном цикле $C_{\nu}$ подстановки $\chi_{A C D B}$, то, заменяя значения $T_{A}$ на $C_{\nu}$ противоположными, получаем трафарет $T_{A}^{\prime}$, накрывающий $\lambda$, для которого выполняется условие $\alpha \in \operatorname{Tr}\left(F^{\prime}, \lambda\right), F^{\prime}=\left(A, B, C, D, T^{\prime}{ }_{A}\right)$. Если же $p_{1} \in C_{\nu}, p_{2} \in C_{\mu}-$ элементы кратности 1 , лежащие на различных циклах $C_{\nu} \neq C_{\mu}$ подстановки $\chi_{A C D B}$, то, заменяя одновременно значения $T_{A}$ на циклах $C_{\nu}$ и $C_{\mu}$ противоположными, также получим трафарет $T_{A}^{\prime}$, накрывающий $\lambda$, для которого выполняется условие $\alpha \in \operatorname{Tr}\left(F^{\prime}, \lambda\right), F^{\prime}=\left(A, B, C, D, T^{\prime}{ }_{A}\right)$. Таким образом всего можно построить $2^{n}$ трафаретов с указанным свойством. Очевидно, что таким способом получаются все такие трафареты. 
Следствие. Пусть $A, B, C, D-Л К$ порядка п $и \lambda \subseteq\{1, \ldots, n\}^{2}-$ nодстановочное 2-сбалансированное мультимножество позииий ЛК А. Пусть $S_{2 n}(\lambda)$ - множество всех подстановок степени $2 n$, порождающих $\lambda$. Tогда $S_{2 n}(\lambda)$ разбивается на такие подмножества $N_{1}, \ldots, N_{t}: S_{2 n}(\lambda)=\bigcup_{i=1}^{t} N_{i}$, $N_{i} \cap N_{j}=\varnothing$ при $i \neq j$, что для любого трафарета $T_{A}$, согласованного с набором $A, B, C, D$, и любой подстановки $\alpha \in S_{2 n}(\lambda)$ из условия $\alpha \in \operatorname{Tr}(F)$, $F=\left(A, B, C, D, T_{A}\right)$, вытекает, что $\operatorname{Tr}(F, \lambda)=N_{i}$, где $i$ определяется условием $\alpha \in N_{i}$.

Доказательство. Пусть для трафарета $T_{A}^{(1)}$ выполняются условия $\alpha, \beta \in \operatorname{Tr}\left(F^{(1)}, \lambda\right), F^{(1)}=\left(A, B, C, D, T_{A}^{(1)}\right), \alpha \neq \beta$. Пусть для другого трафарета $T_{A}^{(2)}$ выполнено $\alpha \in \operatorname{Tr}\left(F^{(2)}, \lambda\right), F^{(2)}=\left(A, B, C, D, T_{A}^{(2)}\right)$. Докажем, что $\beta \in \operatorname{Tr}\left(F^{(2)}, \lambda\right)$.

Перейдем от трафаретов $T_{A}^{(1)}, T_{A}^{(2)}$ к соответствующим им накрывающим $\lambda$ трафаретам $\left(T_{A}^{(1)}\right)^{\prime},\left(T_{A}^{(2)}\right)^{\prime}$. Тогда $\alpha \in \operatorname{Tr}\left(\left(F^{(1)}\right)^{\prime}, \lambda\right)$, $F^{(1)}=\left(A, B, C, D,\left(T_{A}^{(1)}\right)^{\prime}\right)$ и одновременно $\alpha \in \operatorname{Tr}\left(\left(F^{(2)}\right)^{\prime}, \lambda\right), F^{(2)}=$ $=\left(A, B, C, D,\left(T_{A}^{(2)}\right)^{\prime}\right)$, поэтому, в соответствии с п. 2) доказательства утверждения 3 , трафарет $\left(T_{A}^{(2)}\right)^{\prime}$ получается из трафарета $\left(T_{A}^{(1)}\right)^{\prime}$ указанными в этом пункте преобразованиями, и, следовательно, $\beta \in \operatorname{Tr}\left(\left(F^{(2)}\right)^{\prime}, \lambda\right)=\operatorname{Tr}\left(F^{(2)}, \lambda\right)$.

Определение. Пусть $\lambda$ - подстановочное 2-сбалансированное мультимножество позиций ЛК $A$ и $T_{A}=\left(t_{i j}\right)_{n \times n}-$ произвольный трафарет. $B e$ сом трафарета $T_{A}$ относительно $\lambda$ называется $w_{\lambda}\left(T_{A}\right)=\sum_{p \in \lambda} t_{p} \pi_{p}$, где $t_{p}-$ значение $T_{A}$ на позиции $p, \pi_{p}-$ кратность $p$ в $\lambda, \pi_{p} \in\{1,2\}$.

ЗАмЕчАния. 1) Если $T_{A}^{\prime}-$ накрывающий $\lambda$ трафарет, соответствующий трафарету $T_{A}$, то $w_{\lambda}\left(T_{A}\right)=w_{\lambda}\left(T_{A}^{\prime}\right)$.

2) $\varpi_{\lambda}\left(T_{A}\right)=w\left(T_{A} * \Pi_{\lambda}\right)-$ вес трафарета $T_{A}$ относительно $\lambda$ равен весу (сумме элементов) целочисленной матрицы $T_{A} * \Pi_{\lambda}$.

Лемма 2. Пусть $\alpha \subset\{1, \ldots, 2 n\}^{2}-$ подстановка степени $2 n$, порождающая подстановочное 2-сбалансированное мультимножество позиций $\lambda$, $\underline{\lambda} \subseteq\{1, \ldots, n\}^{2}$, в ЛК А и $T_{A}-$ согласованный с набором $A, B, C, D$ трафаpeт. Тогда длины наборов $L_{1}, L_{2}, M_{1}, M_{2}$, построенных для подстановки $\alpha$, имеют одинаковую четность с весом ш $=w_{\lambda}\left(T_{A}\right)$.

Доказательство. В силу замечания 1), сформулированного перед леммой, ее достаточно доказать для трафаретов, накрывающих $\lambda$. Пусть сначала 
$w=w_{\lambda}\left(T_{A}\right)=0$, т. е. $T_{A}=(0)_{n \times n}$, а число позиций подстановки $\alpha$, попадающих в ЛК $A$, равно $t$. Тогда в каждый из ЛК $B$ и $C$ попадает по $n-t$ позиций $\alpha$, а в ЛК $D$ попадает также $t$ позиций. Элементы ЛК $F=\left(A, B, C, D, T_{A}\right)$, находящиеся на позициях $\alpha$ из $A$ и $D$, лежат во множестве $\{n, \ldots, 2 n-1\}$, поэтому номера их строк попадают в набор $L_{2}$. Таким образом, этот набор имеет длину $2 t$. Аналогично, элементы $F$, находящиеся на позициях $\alpha$ в латинских квадратах $B$ и $C$, лежат во множестве $\{0, \ldots, n-1\}$, их номера строк попадают в набор $L_{1}$, который тем самым имеет длину $2 n-2 t$. Итак, при $w=w_{\lambda}\left(T_{A}\right)=0$ лемма доказана.

Пусть теперь $T_{A}^{\prime}$ - произвольный трафарет, накрывающий $\lambda$ и согласованный с набором ЛК $A, B, C, D$. Пусть $w^{\prime}=w_{\lambda}\left(T_{A}^{\prime}\right)$, а $C_{1}, \ldots, C_{s}-$ все те циклы подстановки $\chi_{A C D B}$, которые имеют непустое пересечение с $\lambda$ и на позициях которых в трафарете $T_{A}^{\prime}$ стоят единицы. Переберем по очереди эти циклы и для каждого из них обнулим соответствующие им позиции в $T_{A}^{\prime}$. $\mathrm{B}$ результате получим рассмотренный ранее трафарет $T_{A}=(0)_{n \times n}$. С помощью леммы 1 проследим, что при этом происходит с длинами наборов $L_{1}, L_{2}, M_{1}, M_{2}$. Если очередной цикл $C_{i}, i \in\{1, \ldots, s\}$, содержит элемент $p \in \lambda$ кратности 2 или два элемента $p_{1}, p_{2} \in \lambda, p_{1} \neq p_{2}$, кратностей 1 , то, согласно пп. 2.2 и 2.3 леммы 1, при обнулении элементов трафарета на местах цикла $C_{i}$ не изменяются длины наборов $L_{1}, L_{2}, M_{1}, M_{2}$. При этом вес трафарета уменьшается на 2. Если же очередной $C_{i}, i \in\{1, \ldots, s\}$, содержит единственный элемент $p \in \lambda$ кратности 1, то по п. 2.1 леммы 1 длина одного из наборов $L_{1}$ или $L_{2}$ увеличится на 1 , а другого уменьшится на 1 . При этом вес трафарета уменьшается на 1 . Лемма 2 доказана.

Следствие. Пусть $A, B, C, D-Л К$ порядка $n, \lambda$ - подстановочное 2-сбалансированное мультимножество позищий ЛК $A$ и $T_{A}$ - трафарет, согласованный с набором $A, B, C, D, F=\left(A, B, C, D, T_{A}\right)$, - распавшийся ЛК порядка $2 n$, $=\varpi_{\lambda}\left(T_{A}\right)$. Если числа $n$ и ш имеют различную четность, то $\operatorname{Tr}(F, \lambda)=\varnothing$.

Доказательство. Действительно, в этом случае длины наборов $L_{1}, L_{2}, M_{1}, M_{2}$, построенных для любой подстановки $\alpha$, порождающей $\lambda$, имеют одинаковую с ш четность. А для того, чтобы $\alpha$ была трансверсалью, как указано выше, необходимо равенство этих длин числу $n$.

В качестве итога опишем метод, позволяющий быстро строить множество трансверсалей для любого распавшегося ЛК $F=\left(A, B, C, D, T_{A}\right)$, задаваемого фиксированным набором $A, B, C, D$ ЛК порядка $n$ и произвольным согласованным с этим набором трафаретом $T_{A}$. 
Сначала для заданного набора ЛК $A, B, C, D$ проведем следующую предварительную работу.

1) Перебором всех подстановок $\alpha \in S_{2 n}$ степени $2 n$ построим совокупность $\Lambda=\left\{\lambda_{1}, \ldots, \lambda_{V}\right\}$ всех подстановочных 2-сбалансированных мультимножеств позиций ЛК $A$ и наборы порождающих их подстановок $\left\{S_{2 n}\left(\lambda_{1}\right), \ldots, S_{2 n}\left(\lambda_{V}\right)\right\}$.

2) Для каждого $i \in\{1, \ldots, V\}$ и каждой подстановки $\alpha \in S_{2 n}\left(\lambda_{i}\right)$, в соответствии с утверждением 5 , строим $2^{n}$ накрывающих $\lambda_{i}$ трафаретов $T_{A}$ таких, что $\alpha \in \operatorname{Tr}(F), F=\left(A, B, C, D, T_{A}\right)$.

3) Для каждого $i \in\{1, \ldots, V\}$, в соответствии со следствием к утверждению 5, разобьем множество $S_{2 n}\left(\lambda_{i}\right)$ подстановок, порождающих $\lambda_{i}$, на непересекающиеся подмножества: $S_{2 n}\left(\lambda_{i}\right)=\bigcup_{j=1}^{k_{i}} N_{j}\left(\lambda_{i}\right)$. При этом подстановки $\alpha \in S_{2 n}\left(\lambda_{i}\right)$, у которых в п. 2) получаются одинаковые множества накрывающих $\lambda_{i}$ трафаретов, попадают в одно подмножество $N_{j}\left(\lambda_{i}\right), j \in\left\{1, \ldots, k_{i}\right\}$.

На этом предварительная работа завершается, в результате чего получаются три массива:

1) массив $\Lambda=\left\{\lambda_{1}, \ldots, \lambda_{V}\right\}$ подстановочных 2-сбалансированных мультимножеств позиций ЛК $A$;

2) массив $S_{2 n}(\Lambda)=\bigcup_{i=1}^{V} S_{2 n}\left(\lambda_{i}\right)=\bigcup_{i=1}^{V} \bigcup_{j=1}^{k_{i}} N_{j}\left(\lambda_{i}\right)$ подстановок степени $2 n$;

3) массив $\left\{W_{1}\left(\lambda_{1}\right), \ldots, W_{k_{1}}\left(\lambda_{1}\right), \ldots, W_{1}\left(\lambda_{V}\right), \ldots, W_{k_{V}}\left(\lambda_{V}\right)\right\}$ множеств трафаретов, для которых выполнены условия: $\left|W_{k_{j}}\left(\lambda_{i}\right)\right|=2^{n}(i \in\{1, \ldots, V\}$, $\left.\in\left\{1, \ldots, k_{i}\right\}\right)$, все трафареты из $W_{j}\left(\lambda_{i}\right)$ накрывают $\lambda_{i}$ и множеству трафаретов $W_{k_{j}}\left(\lambda_{i}\right)$ соответствует множество $N_{j}\left(\lambda_{i}\right)$ подстановок степени $2 n$, причем для любого трафарета $T_{A} \in W_{k_{j}}\left(\lambda_{i}\right)$ имеет место равенство $\operatorname{Tr}\left(F, \lambda_{i}\right)=N_{j}\left(\lambda_{i}\right)$, где $F=\left(A, B, C, D, T_{A}\right)$.

Теперь с помощью построенных массивов легко строится множество трансверсалей для любого распавшегося ЛК $F=\left(A, B, C, D, T_{A}\right)$ :

1) Для каждого $i \in\{1, \ldots, V\}$ по трафарету $T_{A}$ строится соответствующий ему трафарет $\left(T_{A}\right)_{\lambda_{i}}^{\prime}$, накрывающий $\lambda_{i}$.

2) Для каждого $i \in\{1, \ldots, V\}$ находим номер $j\left(T_{A}, i\right) \in\left\{1, \ldots, k_{i}\right\}$, удовлетворяющий условию $\left(T_{A}\right)_{\lambda_{i}}^{\prime} \in W_{j\left(T_{A}, i\right)}\left(\lambda_{i}\right)$. Если такой номер $j\left(T_{A}, i\right)$ существует, то $\operatorname{Tr}\left(F, \lambda_{i}\right)=N_{j\left(T_{A}, i\right)}\left(\lambda_{i}\right)$. Если такого номера нет (в частности, это может произойти, если $\varpi_{\lambda_{i}}\left(T_{A}\right)$ и $n$ разной четности), то $\operatorname{Tr}\left(F, \lambda_{i}\right)=\varnothing$.

3) В итоге получаем $\operatorname{Tr}(F)=\bigcup_{i=1}^{V} \operatorname{Tr}\left(F, \lambda_{i}\right)=\bigcup_{i=1}^{V} N_{j\left(T_{A}, i\right)}\left(\lambda_{i}\right)$. 
Автор благодарен Ф. М. Малышеву за полезные замечания по данной работе и, в частности, за формулировку п. 2) следствия к утверждению 3.

\section{Приложение}

Примеры наборов латинских квадратов $A, B, C, D$ порядка 5 с различными числами циклов $k$ в подстановках $\chi_{A C D B}$ :

1) $k \in\{5, \ldots, 25\} \backslash\{24\}, A=B=C=\left(\begin{array}{lllll}0 & 1 & 2 & 3 & 4 \\ 1 & 0 & 3 & 4 & 2 \\ 2 & 3 & 4 & 0 & 1 \\ 3 & 4 & 1 & 2 & 0 \\ 4 & 2 & 0 & 1 & 3\end{array}\right)$,

\begin{tabular}{|c|c|c|c|c|c|c|c|c|c|c|c|c|c|c|c|c|c|c|c|}
\hline & \multicolumn{5}{|c|}{5} & \multicolumn{4}{|c|}{6} & \multicolumn{5}{|c|}{7} & \multicolumn{5}{|c|}{8} \\
\hline & 11 & 0 & 3 & 4 & $2)$ & 71 & 0 & 3 & 4 & 10 & 1 & 3 & 4 & & 10 & 1 & 3 & 4 & \\
\hline & 2 & 3 & 4 & 0 & 1 & 2 & 3 & 4 & 1 & 2 & 3 & 4 & 0 & 1 & & 4 & 1 & 0 & \\
\hline & 3 & 4 & 1 & 2 & 0 & 0 & 4 & 1 & 2 & 3 & 4 & 1 & 2 & 0 & & 2 & 0 & 3 & 4 \\
\hline & 4 & 2 & 0 & 1 & 3 & 4 & 2 & 0 & 31 & 4 & 2 & 0 & 1 & 3 & & 3 & 2 & 1 & 0 \\
\hline & 0 & 1 & 2 & 3 & 4) & 3 & 1 & 2 & $\begin{array}{ll}0 & 4\end{array}$ & 1 & 0 & 2 & 3 & 4) & 3 & 0 & 4 & 2 & 1 \\
\hline & \multirow{2}{*}{\multicolumn{5}{|c|}{ 4) }} & \multicolumn{4}{|c|}{10} & \multicolumn{5}{|c|}{11} & \multicolumn{5}{|c|}{12} \\
\hline & & & & & & 70 & 1 & 2 & 3 & 70 & 1 & 2 & 3 & & 70 & 1 & 2 & 3 & \\
\hline & 2 & 3 & 4 & 1 & 0 & & 3 & 4 & 0 & & 4 & 1 & 0 & 3 & & 0 & 4 & 2 & \\
\hline & 1 & 4 & 0 & $\begin{array}{ll}3 & 2\end{array}$ & 2 & & 4 & 1 & 2 & 3 & 2 & 0 & 4 & 1 & & 4 & 0 & 1 & \\
\hline & 4 & 2 & 3 & 0 & & & 2 & 0 & 1 & 4 & 0 & 3 & 1 & & 4 & 2 & 3 & 0 & \\
\hline & 3 & 0 & & 2 & 4) & & & 3 & 4 & 1 & & & 2 & & & & & & \\
\hline
\end{tabular}

\begin{tabular}{|c|c|c|c|c|c|c|c|c|c|c|c|c|c|c|c|c|c|c|c|c|c|}
\hline$k$ & \multicolumn{5}{|c|}{13} & \multicolumn{5}{|c|}{14} & \multicolumn{5}{|c|}{15} & \multicolumn{6}{|c|}{16} \\
\hline & 70 & 1 & 2 & 3 & & 70 & & 2 & 3 & & & 1 & 2 & 3 & & & & & 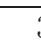 & & \\
\hline & 1 & 2 & 4 & 0 & 3 & 1 & 0 & 4 & 2 & 3 & . & 0 & 3 & 4 & 2 & & & & 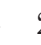 & 2 & \\
\hline$D$ & 2 & 4 & 3 & 1 & 0 & 3 & 4 & 1 & 0 & 2 & 3 & 4 & 1 & 2 & 0 & & & & 3 & & \\
\hline & 4 & 3 & 0 & 2 & 1 & 4 & 2 & 3 & 1 & 0 & , & 2 & 0 & 1 & 3 & & & & & & \\
\hline & 3 & 0 & 1 & 4 & 2) & (2) & 3 & 0 & 4 & & 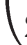 & 3 & 4 & 0 & 1) & & & & & & 0 \\
\hline
\end{tabular}

\begin{tabular}{|c|c|c|c|c|c|c|c|c|c|c|c|c|c|c|c|c|c|c|c|c|}
\hline & \multicolumn{5}{|c|}{17} & \multicolumn{5}{|c|}{18} & \multicolumn{5}{|c|}{19} & \multicolumn{5}{|c|}{20} \\
\hline \multirow{5}{*}{$D$} & 70 & 1 & 2 & 3 & 4) & 0 & 1 & 2 & 3 & & 70 & 1 & 2 & 3 & & 70 & 1 & 2 & 3 & \\
\hline & 1 & 0 & 3 & 4 & 2 & 1 & 0 & 3 & 4 & 2 & 1 & 0 & 3 & 4 & & & 0 & 3 & & \\
\hline & 3 & 4 & 0 & 2 & 1 & 2 & 4 & 0 & 1 & 3 & 2 & 4 & 0 & & & 2 & 3 & 4 & & \\
\hline & 4 & 2 & 1 & 0 & 3 & 3 & 2 & 4 & 0 & 1 & 4 & 3 & 1 & 2 & & 4 & 2 & 0 & & 3 \\
\hline & (2 & 3 & 4 & 1 & 0) & 4 & 3 & 1 & 2 & $0)$ & 3 & 2 & 4 & & 1) & 3 & 4 & 1 & 2 & \\
\hline$k$ & \multicolumn{5}{|c|}{21} & \multicolumn{5}{|c|}{22} & \multicolumn{5}{|c|}{23} & \multicolumn{5}{|c|}{25} \\
\hline \multirow{5}{*}{$D$} & 70 & 1 & 2 & 3 & & 70 & & 2 & 3 & & 70 & 1 & 2 & 34 & & 70 & 1 & 2 & 3 & \\
\hline & & 0 & 3 & 4 & 2 & 1 & 0 & 3 & 4 & 2 & 2 & 0 & 3 & & & & 0 & 3 & & \\
\hline & & 3 & 4 & 1 & 0 & 3 & 2 & 4 & 0 & 1 & 1 & 3 & 4 & 0 & & 2 & 3 & 4 & 0 & \\
\hline & 3 & 4 & 0 & 2 & & 4 & 3 & 1 & 2 & & 3 & 4 & 1 & 2 & & 3 & 4 & 1 & 2 & \\
\hline & 4 & 2 & 1 & & & 2 & & & & & & & 0 & & & & & 0 & & \\
\hline
\end{tabular}


2) Набор латинских квадратов $A, B, C, D$ порядка 5 с 24 циклами в подстановке $\chi_{A C D B}$ :

$$
\begin{gathered}
A=\left(\begin{array}{lllll}
2 & 0 & 3 & 4 & 1 \\
4 & 3 & 1 & 2 & 0 \\
1 & 4 & 0 & 3 & 2 \\
0 & 2 & 4 & 1 & 3 \\
3 & 1 & 2 & 0 & 4
\end{array}\right), \quad B=\left(\begin{array}{llllll}
1 & 2 & 4 & 0 & 3 \\
4 & 3 & 1 & 2 & 0 \\
0 & 1 & 2 & 3 & 4 \\
3 & 4 & 0 & 1 & 2 \\
2 & 0 & 3 & 4 & 1
\end{array}\right), \\
C=\left(\begin{array}{lllll}
4 & 2 & 3 & 0 & 1 \\
1 & 4 & 0 & 2 & 3 \\
3 & 1 & 2 & 4 & 0 \\
2 & 0 & 1 & 3 & 4 \\
0 & 3 & 4 & 1 & 2
\end{array}\right), \quad D=\left(\begin{array}{lllll}
0 & 1 & 2 & 3 & 4 \\
1 & 4 & 0 & 2 & 3 \\
3 & 0 & 4 & 1 & 2 \\
2 & 3 & 1 & 4 & 0 \\
4 & 2 & 3 & 0 & 1
\end{array}\right) .
\end{gathered}
$$

\section{Список литературы}

1. Сачков В.Н. Введение в комбинаторные методы дискретной математики. - М.: МЦНМО, 2004.

2. Wanless I. M. A generalisation of transversals for Latin squares // Electron. J. Comb. - 2002. - V. 9. - P. 12.

3. Wanless I.M. Transversals in Latin squares // In: Quasigroups and Related Systems. - Inst. of Mathematics, Academy of Sciences of Moldova. 2007. - V. 15. - P. 169-190.

4. McKay B.D., Meynert A., Myrvold W.J. Small Latin squares, quasigroups and loops // J. Combin. Des. - 2007. - V. 15. № 2. - P. 98-119. 